DOI: 10.12957/demetra.2018.32295

\title{
Qualidade de vida de crianças e adolescentes com fibrose cística: importância da imagem corporal e impacto do estado nutricional, idade e raça/cor na percepção dos pacientes e responsáveis
}

\author{
Quality of life of children and adolescents with cystic fibrosis: the importance of body image and \\ the impact of nutritional status, age and race/skin color on patients' and caregivers' perceptions
}

Laura Andrade da Silva'

Ana Carla Pazini Lima'

Veronica Lourenço Wittmer'

Fernanda Mayrink Gonçalves Liberato ${ }^{2}$

Luana da Silva Baptista Arpini²

Flávia Marini Paro ${ }^{7}$

' Universidade Federal do Espírito Santo, Departamento de Educação Integrada em Saúde. Vitória, ES, Brasil.

${ }^{2}$ Hospital Infantil Nossa Senhora da Glória. Vitória, ES, Brasil.

Correspondência / Correspondence

Flávia Marini Paro

Universidade Federal do Espírito Santo, Centro de Ciências da Saúde, Departamento de Educação Integrada em Saúde, Av. Maruípe, 1468, VitóriaES, Brasil. CEP: 29043-900.

E-mail: flamarp@yahoo.com

\section{Resumo}

Objetivo: Identificar os principais fatores relacionados à qualidade de vida de crianças e adolescentes com fibrose cística. Métodos: Estudo transversal, com indivíduos com fibrose cística, entre 7 e 18 anos, de um Centro de Referência Estadual. A qualidade de vida foi avaliada com o Cystic Fibrosis Questionnaire. Testes usados na análise estatística: Mann-Whitney e Correlação de Spearman. Nível de significância adotado: 5\%. Resultados: Na percepção dos pacientes, os domínios da qualidade de vida com piores médias foram imagem corporal $(64,3 \pm 29,6)$ e peso $(47,6 \pm 42,4)$. Na percepção dos responsáveis, peso também apresentou a pior média $(52,8 \pm 43,7)$. O impacto dos tratamentos na qualidade de vida foi maiora partir dos 14 anos $(p=0,012)$, e o impacto da alimentação foi maior entre menores de 14 anos $(\mathrm{p}=0,04)$. Indivíduos brancos apresentaram melhores escores do que negros/pardos no domínio imagem corporal $(\mathrm{p}=0,049)$. Houve correlação positiva moderada entre PIMC/I e o domínio respiratório e correlação positiva forte entre $\mathrm{PIMC} / \mathrm{I}$ e imagem corporal. Conclusões: A correlação entre pIMC/I e imagem corporal sugere que o baixo-peso afeta negativamente a imagem corporal, o que merece atenção, pois imagem corporal e peso foram os domínios com piores pontuações na percepção dos pacientes, sendo que o peso também teve a pior pontuação na percepção dos responsáveis. Além disso, o baixo-peso aumentou o impacto dos sintomas respiratórios na qualidade de vida. $\mathrm{O}$ impacto dos tratamentos aumentou com a idade, enquanto o da 
alimentação diminuiu com a idade. Também se observou impacto da raça/cor no domínio imagem corporal da qualidade de vida.

Palavras-chave: Fibrose cística. Imagem corporal. Avaliação nutricional. Índice de massa corporal. Qualidade de vida. Desnutrição.

\section{Abstract}

Objective: To identify factors related to the quality of life of children and adolescents with cystic fibrosis. Methods: Crosssectional study of patients with cystic fibrosis, aged 7-18 years, from a Pediatric Referral Center in Brazil. The Cystic Fibrosis Questionnaire was used to evaluate the quality of life. The MannWhitney Test and the Spearman Correlation Test were used for the statistical analysis. The significance level adopted was 5\%. Results: The domains with the lowest averages were body image

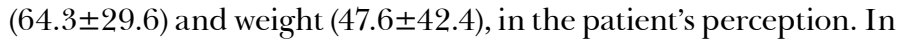
the parents' perception, weight also presented the lowest average $(52.8 \pm 43.7)$. The treatment domain had greater impact on the quality of life of patients aged 14 years $(\mathrm{p}=0.012)$ and older, and eating had greater impact among children younger than 14 years $(p=0.04)$. The white individuals had better scores than the black/brown individuals in the body image domain $(\mathrm{p}=0.049)$. A moderate positive correlation was found between $\mathrm{pBMI} / \mathrm{A}$ and the respiratory domain and a strong positive correlation between pBMI/A and body image. Conclusions: The correlation between pBMI/A and body image suggests that low weight negatively affects body image, which deserves attention, since body image and weight were the domains with the lowest scores in the patient's perception, and weight also had the lowest score in the perception of those responsible for the patients. In addition, low weight increased the impact of respiratory symptoms on the quality of life. The impact of treatments was greater in older patients, while the impact of eating was greater in younger patients. Race/skin color had an impact on the body image domain.

Keywords: Cystic fibrosis. Body image. Nutrition assessment. Body mass index. Quality of life. Malnutrition. 


\section{Introdução}

A fibrose cística (FC) é uma doença genética, autossômica recessiva, crônica e progressiva, que tem como causa mutações em um gene localizado no braço longo do cromossomo 7.,2 Essas mutações interferem na regulação do transporte iônico, tendo como consequências a desidratação das secreções mucosas e o aumento da sua viscosidade, favorecendo a obstrução dos ductos das glândulas exócrinas, acompanhada de reações inflamatórias, sendo, portanto, uma doença multissistêmica. ${ }^{3}$ As características fenotípicas, os sistemas acometidos e a gravidade da doença variam de acordo com o tipo de mutação. ${ }^{4}$

Embora o acometimento do sistema respiratório seja a principal causa de morbidade e mortalidade na doença, ${ }^{3,4}$ também é muito importante o comprometimento gastrointestinal, devido à obstrução dos ductos pancreáticos e biliares. ${ }^{4}$ Nos casos mais graves, a obstrução dos ductos pancreáticos por muco tem início desde a vida intrauterina, e os pacientes apresentam insuficiência pancreática ao nascer ou pouco tempo após o nascimento. ${ }^{4}$ Indivíduos com mutações menos severas podem, ou não, desenvolver pancreatite obstrutiva crônica e insuficiência pancreática, ao longo da vida. ${ }^{4}$ A obstrução dos ductos pancreáticos prejudica a liberação das enzimas para o duodeno, o que causa má digestão e má absorção de gorduras, proteínas e carboidratos, sendo frequentes diarreias crônicas, com fezes volumosas e gordurosas, e desnutrição calórica proteica. ${ }^{3}$

Os avanços no conhecimento da FC, seu diagnóstico precoce e a evolução do tratamento, com novos medicamentos e ênfase na atuação da equipe multidisciplinar, proporcionaram aumento da expectativa de vida desta população. ${ }^{4}$ Dados demográficos norte-americanos revelam que, pela primeira vez, há mais adultos do que crianças com a doença. ${ }^{5}$

É fundamental que a melhora da qualidade de vida (QV) acompanhe o aumento da longevidade. ${ }^{6}$ Por essa razão, instrumentos específicos para a avaliação da QV de indivíduos com FC têm sido desenvolvidos e aperfeiçoados. ${ }^{7,8}$

Questionários de avaliação da QV fornecem de forma padronizada, válida e confiável, a perspectiva dos pacientes e familiares quanto aos benefícios e limitações de uma intervenção, informações que não podem ser obtidas nas análises de outros desfechos clínicos. ${ }^{9}$ Além disso, avaliações rotineiras da $\mathrm{QV}$ e registros longitudinais permitem identificar e monitorar as dificuldades vivenciadas pelos pacientes ao longo do tempo, ${ }^{10}$ contribuindo para análises técnicas e para a elaboração de políticas de saúde específicas. ${ }^{11}$

Os fatores que afetam negativamente a QV na FC têm sido estudados, ${ }^{10}$ mas são necessários mais conhecimentos para que se possa planejar políticas públicas com impacto neste desfecho. Assim este estudo teve como objetivo identificar os principais fatores relacionados à QV de crianças e adolescentes com FC. 


\section{Métodos}

Estudo transversal, aprovado pelo Comitê de Ética em Pesquisa da Universidade Federal do Espírito Santo (UFES) (Parecer n⿳ 352/572, CAAE: 16947313.1.0000.5060), realizado com pacientes com fibrose cística (FC) atendidos entre novembro de 2015 e agosto de 2016 no Hospital Estadual Infantil Nossa Senhora da Glória (HINSG), único centro de referência estadual infantil para o tratamento da FC no Estado do Espírito Santo (ES), Brasil.

Os critérios de inclusão foram todos pacientes diagnosticados com FC, com idade entre 7 e 18 anos, cadastrados e em tratamento no HINSG. Os critérios de exclusão foram: recusa do paciente e/ou responsável em participar; pacientes que não apresentavam condição cognitiva que permitisse interação com o examinador e o não comparecimento do paciente às avaliações durante o período da coleta de dados.

O total de pacientes cadastrados e atendidos no HINSG era 49 no período do estudo, sendo excluídos 30, devido aos seguintes critérios: 27 (não compareceram nos dias das avaliações), 2 (autismo), 1 (neuropatia). A amostra de conveniência foi composta por 19 pacientes.

A avaliação teve início após a assinatura do Termos de Consentimento Livre e Esclarecido e do Termo de Assentimento, quando necessário. Informações sobre dados pessoais, idade, bactérias de colonização, tempo de colonização, tabagismo passivo ou ativo foram perguntadas aos pacientes/ responsáveis e confirmadas nos prontuários.

Para classificar os pacientes quanto à gravidade, foi usado o Escore de Shwachman $(<40=$ grave; $41-55$ = moderado; $56-70=$ médio; $71-85=$ bom; e $86-100=$ excelente) ${ }^{12}$

Na avaliação nutricional, foi calculado o índice de massa corporal (IMC) para a determinação do percentil do índice de massa corporal para idade (pIMC/I), utilizando o software WHO Anthro Plus, 2007 versão 1.0.4. O estado nutricional foi classificado de acordo com o recomendado pela Organização Mundial da Saúde. ${ }^{13}$

Foi aplicado o Cystic Fibrosis Questionnaire (CFQ), traduzido e validado para a língua portuguesa em 2006. ${ }^{14}$ Foram aplicadas as quatro versões do CFQ em português: CFQ para grupos de 6 a 11 anos; CFQ para grupos de 12 a 13 anos; CFQ maiores de 14 anos; CFQ para os pais/responsáveis das crianças de 6 a 13 anos. São 35 questões divididas em oito domínios (físico, emocional, social, imagem corporal, alimentação, tratamento, respiratório e digestivo) para os dois grupos de 6 a 11 anos e de 12 a 13 anos; 50 questões divididas em 12 domínios (papel social, vitalidade, peso, saúde e os domínios previamente citados) para os maiores de 14 anos e 44 questões divididas em 12 domínios (físico, emocional, social, papel social, alimentação, tratamentos, respiratório, digestivo, vitalidade, saúde, imagem corporal e peso) para os pais/responsáveis. A pontuação de cada domínio varia de 0 a 100. Valores mais altos expressam melhor QV. 
Para o grupo de 7 a 11 anos, o questionário foi aplicado por um entrevistador com auxílio de cartões resposta. Os outros questionários foram preenchidos pelos próprios pacientes e responsáveis, tomando cuidado para não haver influência nas repostas entre ambos.

Para a análise estatística, foi utilizado o software Statistical Package for Social Sciences (IBM SPSS Statistics 22, IBM, Armonk, NY, Estados Unidos). O teste de Mann-Whitney foi usado para avaliar as diferenças entre os grupos, devido à rejeição da hipótese de normalidade. Para analisar as correlações entre os domínios da QV e os valores do Escore de Shwachman e do IMC, foi utilizada a correlação de Spearman. A força da correlação foi avaliada pelo valor do coeficiente de correlação $(\rho)$, de acordo com o seguinte critério: se $0<\rho<0,3$, existe fraca correlação; se 0,3 $\leq \rho<0,6$, existe moderada correlação; se $0,6 \leq \rho<0,9$, existe forte correlação; se $0,9 \leq \rho<1$, existe correlação muito forte..$^{15}$ Foi adotado o nível de significância de $5 \%$.

\section{Resultados}

A média de idade dos pacientes foi de 12,21 \pm 3,78 anos. As características demográficas e clínicas que predominaram na amostra foram: sexo masculino (63,2\%), caucasianos $(68,4 \%)$, não tabagistas passivos $(73,74 \%)$, colonizados por bactérias $(73,7 \%)$, com classificação "bom" no Escore de Shwachman $(57,9 \%)$ e eutróficos $(89,5 \%)$. (tabela 1).

Entre os 14 pacientes colonizados, oito eram colonizados por Staphylococcus aureus (57,14\%) e seis por Pseudomonas aeruginosa (42,85\%), com tempo de colonização variando de 1 a 6 anos (média de 3,14 $\pm 1,9$ anos).

A média de pontuação no Escore Shwachman foi de 78,16 \pm 10,30.

A caracterização da amostra com relação ao pIMC/I está apresentada na tabela 2.

Todos os participantes frequentavam a escola e recebiam atendimento da equipe interdisciplinar, incluindo fisioterapeuta e nutricionista.

No grupo de crianças com idades entre 7 e 13 anos, as melhores e as piores médias nos

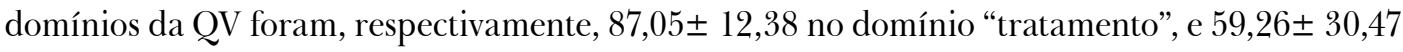
no domínio "imagem corporal". No grupo formado por crianças com 14 anos ou mais, a melhor média foi no domínio "alimentação" $(95,24 \pm 12,59)$, enquanto a pior média foi no domínio "peso" $(47,61 \pm 42,42)$. No geral, o domínio que apresentou a melhor média foi "alimentação" $(83,64 \pm$ $19,01)$ e os domínios com piores médias foram "peso" (47,61 $\pm 42,42)$ e "imagem corporal" $(64,33 \pm$ 29,55) (tabela 3). 
Tabela 1. Caracterização demográfica e clínica dos pacientes com FC segundo a faixa etária. Vitória-ES, 2015/2016.

\begin{tabular}{|c|c|c|c|}
\hline \multirow{2}{*}{ Variáveis } & 7-13 anos & $\geq 14$ anos & Total \\
\hline & $\mathrm{n}(\%)$ & $\mathrm{n}(\%)$ & $\mathrm{n}(\%)$ \\
\hline \multicolumn{4}{|l|}{ Sexo } \\
\hline Masculino & $8(66,7)$ & $4(57,1)$ & $12(63,2)$ \\
\hline Feminino & $4(33,3)$ & $3(42,9)$ & $7(36,8)$ \\
\hline \multicolumn{4}{|l|}{ Raça / Cor } \\
\hline Branco & $9(75,0)$ & $4(57,1)$ & $13(68,4)$ \\
\hline Negro / Pardo & $3(25,0)$ & $3(42,9)$ & $6(31,6)$ \\
\hline \multicolumn{4}{|l|}{ Tabagismo passivo? } \\
\hline Sim & $3(25,0)$ & $2(28,6)$ & $5(26,3)$ \\
\hline Não & $9(75,0)$ & $5(71,4)$ & $14(73,7)$ \\
\hline \multicolumn{4}{|l|}{ Colonizado? } \\
\hline Sim & $9(75,0)$ & $5(71,4)$ & $14(73,7)$ \\
\hline Não & $3(25,0)$ & $2(28,6)$ & $5(26,3)$ \\
\hline \multicolumn{4}{|c|}{ Escore de Shwachman } \\
\hline Excelente (86-100) & $2(16,7)$ & $1(14,3)$ & $3(15,8)$ \\
\hline Bom (71-85) & $7(58,3)$ & $4(57,1)$ & $11(57,9)$ \\
\hline Leve (56-70) & $2(16,7)$ & $2(28,6)$ & $4(21,0)$ \\
\hline Moderado (41-55) & $1(8,3)$ & - & $1(5,3)$ \\
\hline Grave $(<40)$ & - & - & - \\
\hline \multicolumn{4}{|c|}{ Estado Nutricional (IMC/I) } \\
\hline Eutrófico & $10(83,3)$ & $7(100)$ & $17(89,5)$ \\
\hline Magreza & $1(8,3)$ & - & $1(5,25)$ \\
\hline Magreza acentuada & $1(8,3)$ & - & $1(5,25)$ \\
\hline Total & $12(100)$ & $7(100)$ & $19(100)$ \\
\hline
\end{tabular}

FC, fibrose císitica; IMC/I: índice de massa corporal para a idade. 
Tabela 2. Caracterização dos indivíduos com FC com relação ao pIMC/I. Vitória-ES, 2015/2016.

\begin{tabular}{cccccc}
\hline Idade (anos) & Variáveis & $\mathrm{n}$ & Mediana & Média & Desvio-padrão \\
\cline { 2 - 5 } $7-13$ & $\mathrm{pIMC} / \mathrm{I}$ & 12 & 18,45 & 34,37 & 32,38 \\
$\geq 14$ & $\mathrm{pIMC} / \mathrm{I}$ & 7 & 30,80 & 30,31 & 24,51 \\
Total & $\mathrm{pIMC} / \mathrm{I}$ & 19 & 21,80 & 32,88 & 29,07 \\
\hline
\end{tabular}

FC: fibrose cística; pIMC/I: percentil do índice de massa corporal para a idade.

Tabela 3. Pontuação nos domínios da qualidade de vida do Cystic Fibrosis Questionnaire na percepção das crianças/adolescentes e de seus responsáveis. Vitória-ES, 2015/2016.

\begin{tabular}{|c|c|c|c|c|c|}
\hline Grupos & Domínios & $\mathrm{N}$ & $<$ valor & >valor & Média $\pm d p$ \\
\hline \multirow{8}{*}{ 7-13 anos } & Físico & 12 & 33,3 & 100,0 & $67,6 \pm 18,8$ \\
\hline & Emocional & 12 & 41,7 & 91,7 & $73,4 \pm 15,0$ \\
\hline & Social & 12 & 38,1 & 100,0 & $70,2 \pm 16,6$ \\
\hline & Alimentação & 12 & 44,4 & 100,0 & $76,9 \pm 19,2$ \\
\hline & Tratamentos & 12 & 66,7 & 100,0 & $87,1 \pm 12,4$ \\
\hline & Respiratório & 12 & 41,7 & 100,0 & $61,1 \pm 18,2$ \\
\hline & Digestivo & 12 & 0,0 & 100,0 & $63,9 \pm 33,2$ \\
\hline & Imagem Corporal & 12 & 0,0 & 100,0 & $59,3 \pm 30,5$ \\
\hline \multirow{12}{*}{$\geq 14$ anos } & Físico & 7 & 54,2 & 95,8 & $79,2 \pm 14,4$ \\
\hline & Emocional & 7 & 60,0 & 100,0 & $80,9 \pm 16,5$ \\
\hline & Social & 7 & 55,6 & 94,4 & $73,1 \pm 16,1$ \\
\hline & Alimentação & 7 & 66,7 & 100,0 & $95,2 \pm 12,6$ \\
\hline & Tratamentos & 7 & 0.0 & 77,8 & $61,9 \pm 28,6$ \\
\hline & Respiratório & 7 & 50,0 & 88,9 & $71,4 \pm 12,2$ \\
\hline & Digestivo & 7 & 66,7 & 100,0 & $85,7 \pm 13,9$ \\
\hline & Vitalidade & 7 & 25,0 & 91,7 & $66,7 \pm 24,5$ \\
\hline & Saúde & 7 & 22,2 & 88,9 & $77,8 \pm 24,9$ \\
\hline & Imagem Corporal & 7 & 33,3 & 100,0 & $73,0 \pm 27,9$ \\
\hline & Papel Social & 7 & 41,7 & 100,0 & $81,0 \pm 20,8$ \\
\hline & Peso & 7 & 0,0 & 100,0 & $47,6 \pm 42,4$ \\
\hline
\end{tabular}




\begin{tabular}{|c|c|c|c|c|c|}
\hline Grupos & Domínios & $\mathrm{N}$ & $<$ valor & >valor & Média $\pm \mathrm{dp}$ \\
\hline \multirow{12}{*}{$\begin{array}{l}\text { Total } \\
\text { (crianças e } \\
\text { adolescentes) }\end{array}$} & Físico & 19 & 33,3 & 100,0 & $71,9 \pm 17,8$ \\
\hline & Emocional & 19 & 41,7 & 100,0 & $76,2 \pm 15,6$ \\
\hline & Social & 19 & 38,1 & 100,0 & $71,3 \pm 16,0$ \\
\hline & Alimentação & 19 & 44,4 & 100,0 & $83,6 \pm 19,0$ \\
\hline & Tratamentos & 19 & 0,0 & 100,0 & $77,8 \pm 22,8$ \\
\hline & Respiratório & 19 & 41,7 & 100,0 & $64,9 \pm 16,7$ \\
\hline & Digestivo & 19 & 0,0 & 100,0 & $72,0 \pm 29,3$ \\
\hline & Vitalidade & 7 & 25,0 & 91,7 & $66,7 \pm 24,5$ \\
\hline & Saúde & 7 & 22,2 & 88,9 & $77,8 \pm 24,9$ \\
\hline & Imagem Corporal & 19 & 0,0 & 100,0 & $64,3 \pm 29,6$ \\
\hline & Papel Social & 7 & 41,7 & 100,0 & $81,0 \pm 20,8$ \\
\hline & Peso & 7 & 0,0 & 100,0 & $47,6 \pm 42,4$ \\
\hline \multirow{11}{*}{$\begin{array}{l}\text { Responsáveis } \\
\text { (das crianças } \\
\text { de } 7 \text { a } 13 \\
\text { anos) }\end{array}$} & Físico & 12 & 20,8 & 95,8 & $66,6 \pm 20,5$ \\
\hline & Emocional & 12 & 53,3 & 100,0 & $85,0 \pm 14,5$ \\
\hline & Social & 12 & 8,3 & 100,0 & $68,8 \pm 26,2$ \\
\hline & Alimentação & 12 & 0,0 & 100,0 & $66,7 \pm 35,5$ \\
\hline & Tratamentos & 12 & 0,0 & 88,9 & $60,2 \pm 26,6$ \\
\hline & Respiratório & 12 & 7,0 & 100,0 & $59,4 \pm 27,8$ \\
\hline & Digestivo & 12 & 22,2 & 100,0 & $73,2 \pm 22,5$ \\
\hline & Vitalidade & 12 & 40,0 & 93,3 & $68,3 \pm 18,2$ \\
\hline & Saúde & 12 & 0,0 & 100,0 & $61,6 \pm 33,3$ \\
\hline & Imagem Corporal & 12 & 44,4 & 100,0 & $81,5 \pm 19,7$ \\
\hline & Peso & 12 & 0,0 & 100,0 & $52,8 \pm 43,7$ \\
\hline
\end{tabular}


Na perspectiva dos pais, o domínio que apresentou a melhor média foi "emocional" $(84,98 \pm$ $14,54)$ enquanto o domínio com a média mais baixa foi "peso" (52,78 443,71$)$ (tabela 4).

Na comparação estatística dos escores da QV entre as faixas etárias, o grupo com idade de 14 a 18 anos apresentou valores mais altos no domínio "alimentação" $(\mathrm{p}=0,04)$, do que o grupo com idade entre 7 a 13 anos. Por outro lado, no domínio "tratamento", as crianças de 7 a 13 anos apresentaram valores mais elevados $(\mathrm{p}=0,012)$ (tabela 4$)$.

Quando comparada a percepção dos responsáveis sobre a QV dos seus filhos, com a percepção das próprias crianças sobre a sua QV, foi encontrada diferença significativa apenas no domínio "tratamentos", no qual as crianças apresentaram escores maiores que seus responsáveis $(p=0,003)$ (tabela 4). 
Tabela 4. Escores dos domínios de QV e comparações entre os diferentes grupos etários, e entre as crianças com FC de 7 a 13 anos e seus responsáveis. Vitória-ES, 2015/2016.

\begin{tabular}{|c|c|c|c|c|c|c|}
\hline \multirow{2}{*}{ Domínios } & \multicolumn{3}{|c|}{ Comparação entre faixas etárias } & \multicolumn{3}{|c|}{ Comparação: crianças / responsáveis } \\
\hline & Idade* $^{*}(\mathrm{n})$ & & $\mathrm{p}$ & Grupos (n) & & $\mathrm{p}$ \\
\hline \multirow[t]{2}{*}{ Físico } & $7-13(12)$ & $67,59 \pm 18,77$ & \multirow{2}{*}{0,203} & Crianças (12) & $67,59 \pm 18,77$ & \multirow{2}{*}{0,799} \\
\hline & $\geq 14(7)$ & $79,17 \pm 14,41$ & & Responsáveis (12) & $66,62 \pm 20,49$ & \\
\hline \multirow[t]{2}{*}{ Emocional } & $7-13(12)$ & $73,42 \pm 14,99$ & \multirow{2}{*}{0,611} & Crianças (12) & $73,42 \pm 14,99$ & \multirow{2}{*}{0,060} \\
\hline & $\geq 14(7)$ & $80,94 \pm 16,52$ & & Responsáveis (12) & $84,98 \pm 14,54$ & \\
\hline \multirow[t]{2}{*}{ Social } & $7-13(12)$ & $70,24 \pm 16,63$ & \multirow{2}{*}{0,966} & Crianças (12) & $70,24 \pm 16,63$ & \multirow{2}{*}{0,887} \\
\hline & $\geq 14(7)$ & $73,09 \pm 16,09$ & & Responsáveis (12) & $68,75 \pm 26,15$ & \\
\hline \multirow[t]{2}{*}{ Alimentação } & $7-13(12)$ & $76,87 \pm 19,22$ & \multirow{2}{*}{$0,042^{1}$} & Crianças (12) & $76,87 \pm 19,22$ & \multirow{2}{*}{0,932} \\
\hline & $\geq 14(7)$ & $95,24 \pm 12,59$ & & Responsáveis (12) & $66,67 \pm 35,52$ & \\
\hline \multirow[t]{2}{*}{ Tratamentos } & $7-13(12)$ & $87,05 \pm 12,37$ & \multirow{2}{*}{$0,012^{1}$} & Crianças (12) & $87,05 \pm 12,37$ & \multirow{2}{*}{0,003} \\
\hline & $\geq 14(7)$ & $61,93 \pm 28,59$ & & Responsáveis (12) & $60,20 \pm 26,59$ & \\
\hline \multirow[t]{2}{*}{ Respiratório } & $7-13(12)$ & $61,12 \pm 18,22$ & \multirow{2}{*}{0,148} & Crianças (12) & $61,12 \pm 18,22$ & \multirow{2}{*}{0,977} \\
\hline & $\geq 14(7)$ & $71,36 \pm 12,23$ & & Responsáveis (12) & $59,39 \pm 27,82$ & \\
\hline \multirow[t]{2}{*}{ Digestivo } & 7-13 (12) & $63,91 \pm 33,21$ & \multirow{2}{*}{0,124} & Crianças (12) & $63,91 \pm 33,21$ & \multirow{2}{*}{0,478} \\
\hline & $\geq 14(7)$ & $85,73 \pm 13,91$ & & Responsáveis (12) & $73,16 \pm 22,45$ & \\
\hline \multirow[t]{2}{*}{ Imagem Corporal } & $7-13(12)$ & $59,26 \pm 30,47$ & \multirow[t]{2}{*}{0,302} & Crianças (12) & $59,26 \pm 30,47$ & \multirow{2}{*}{0,052} \\
\hline & $\geq 14(7)$ & $73,01 \pm 27,87$ & & Responsáveis (12) & $81,49 \pm 19,72$ & \\
\hline \multirow[t]{2}{*}{ Vitalidade } & $7-13(0)$ & & & & & \\
\hline & $\geq 14(7)$ & $66,67 \pm 24,54$ & & Responsáveis (12) & $68,33 \pm 18,22$ & \\
\hline \multirow[t]{2}{*}{ Saúde } & $7-13(0)$ & & & & & \\
\hline & $\geq 14(7)$ & $77,79 \pm 24,86$ & & Responsáveis (12) & $61,59 \pm 33,29$ & \\
\hline \multirow[t]{2}{*}{ Peso } & $7-13(0)$ & & & & - & \\
\hline & $\geq 14(7)$ & $47,61 \pm 42,42$ & & Responsáveis (12) & $52,78 \pm 43,72$ & \\
\hline \multirow[t]{2}{*}{ Papel Social } & $7-13(0)$ & & & & & \\
\hline & $\geq 14(7)$ & $80,96 \pm 20,80$ & & & & \\
\hline
\end{tabular}

${ }^{1}$ Diferença estatisticamente significativa pelo teste de teste de Mann-Whitney ( $<$ 0,05); QV: qualidade de vida; FC: fibrose cística; dp: desvio padrão. 
Com relação a raça/cor, os indivíduos brancos $(\mathrm{n}=13$; média $=72,65 \pm 31,64)$ apresentaram melhores escores do que os indivíduos negros/pardos $(n=6$; média $=46,28 \pm 13,02)$ no domínio “imagem corporal” ( $p=0,049)$. Os outros domínios não mostraram diferenças entre esses grupos.

Não foi observada relação dos domínios da QV com tabagismo passivo ou colonização por bactérias. Foi encontrada correlação positiva com força moderada entre o IMC/I e o domínio "respiratório"; e correlação positiva forte entre IMC/I e o domínio “imagem corporal”. (tabela 5). Logo, quanto maior o valor de IMC/I dos indivíduos, maiores também os escores observados nos domínios "respiratório" e "imagem corporal".

Não foi observada correlação do Escore Shwachman com nenhum dos domínios da QV (tabela 6).

Tabela 5. Correlação entre os domínios QV e as escalas de IMC/I das crianças e adolescentes com FC. Vitória-ES, 2015/2016.

\begin{tabular}{lcc}
\hline \multirow{2}{*}{ Qualidade de vida } & \multicolumn{2}{c}{$\mathrm{pIMC} / \mathrm{I}$} \\
\cline { 2 - 3 } Físico & $\rho$ & $\mathrm{p}$-valor \\
Emocional & 0,280 & 0,245 \\
Social & 0,069 & 0,780 \\
Alimentação & 0,078 & 0,752 \\
Tratamentos & 0,270 & 0,263 \\
Respiratório & $-0,134$ & 0,584 \\
Digestivo & $0,493^{1}$ & 0,032 \\
Vitalidade & 0,068 & 0,783 \\
Saúde & 0,288 & 0,531 \\
Imagem Corporal & $-0,178$ & 0,702 \\
Papel Social & $0,683^{1}$ & 0,001 \\
Peso & 0,200 & 0,667 \\
\hline
\end{tabular}

${ }^{1}$ estatisticamente significativo pela correlação de Spearman; QV: qualidade de vida; FC: fibrose cística; IMC/I: índice de massa corporal para a idade; $\rho$ : coeficiente de correlação; pIMC/I: percentil do índice de massa corporal para a idade. 
Tabela 6. Correlação entre os domínios QV e o Escore de Shwachman. Vitória-ES, 2015/2016.

\begin{tabular}{lcc}
\hline \multirow{2}{*}{ Qualidade de vida } & \multicolumn{2}{c}{ Escore Shwachman } \\
\cline { 2 - 3 } Físico & $\rho$ & p-valor \\
Emocional & 0,095 & 0,699 \\
Social & $-0,424$ & 0,071 \\
Alimentação & $-0,022$ & 0,927 \\
Tratamentos & 0,129 & 0,598 \\
Respiratório & 0,065 & 0,790 \\
Digestivo & 0,388 & 0,101 \\
Vitalidade & $-0,172$ & 0,481 \\
Saúde & $-0,312$ & 0,496 \\
Imagem Corporal & 0,327 & 0,474 \\
Papel Social & 0,314 & 0,191 \\
Peso & $-0,191$ & 0,682 \\
\hline
\end{tabular}

Correlação de Spearman; QV: qualidade de vida; $\rho$ : Coeficiente de Correlação

\section{Discussão}

A média geral de pontuação no CFQ foi 71,25, semelhante à média observada em outro estudo, realizado em pacientes clinicamente estáveis. ${ }^{16}$ Não há, porém, valores de referência estabelecidos na literatura. Na Holanda, o CFQ (com exceção do domínio tratamento) foi aplicado em 478 indivíduos saudáveis, na faixa etária de 6 a 20 anos, evidenciando que mesmo crianças e adolescentes saudáveis podem não apresentar pontuações máximas, pois apenas 34,8\% dos indivíduos alcançaram o escore máximo nos domínios avaliados. ${ }^{17}$

Os domínios que apresentaram pior média geral foram "peso" e imagem corporal”, o que significa que estes foram os dois domínios com impacto mais negativo na QV desses indivíduos. A baixa pontuação no domínio "imagem corporal” também foi observada em outro estudo de pacientes com FC. ${ }^{16}$ Por outro lado, crianças e adolescentes saudáveis apresentaram pontuação mais elevada neste domínio em todas as faixas etárias. ${ }^{15} \mathrm{~A}$ FC pode afetar a aparência física, pelo retardo no crescimento, dificuldade de ganho de peso e atraso no desenvolvimento puberal, ${ }^{18}$ 
secundários à desnutrição energético-proteica, o que contribui para o impacto negativo da doença nos domínios "peso" e "imagem corporal”.

A idade teve impacto em dois domínios da QV, alimentação e tratamentos. Adolescentes com idade a partir de 14 anos apresentaram pontuação maior no domínio "alimentação", em comparação com o grupo de 7 a 13 anos. Além disso, o domínio "alimentação" $(95,2 \pm 12,6)$ foi o que apresentou a maior média entre as crianças maiores que 14 anos; e o domínio "peso" apresentou a média mais baixa $(47,6 \pm 42,4)$. Esses achados corroboram outro estudo brasileiro, realizado no estado de São Paulo, no qual também foram observadas maiores e menores pontuações desses domínios nessa faixa etária. ${ }^{19}$ Evidências têm mostrado a preferência dos adolescentes por alimentos processados, com elevado teor calórico, ${ }^{20,21}$ considerados mais prazerosos do que alimentos mais saudáveis. ${ }^{21}$ Em contraposição, também é observada entre adolescentes a cobrança por autocontrole na alimentação, associada à frequente tentativa de restringir o consumo desses alimentos que lhes proporcionam satisfação, para se adequar aos padrões estéticos. ${ }^{21}$ É possível que a alta pontuação dos adolescentes com FC no domínio "alimentação" esteja relacionada à percepção de sua liberdade para consumir alimentos calóricos, que lhes dão prazer, sem as restrições impostas pelo risco de ganho de peso que outros adolescentes de seu convívio enfrentam. Por outro lado, dependendo da gravidade de seu comprometimento digestivo, o adolescente com FC precisará fazer a reposição das enzimas disgestivas $^{4}$ a cada refeição, o que contribui para o impacto negativo do domínio "tratamentos" na sua percepção da QV.

O grupo na faixa etária de 14 a 18 anos apresentou pontuação significativamente menor no domínio "tratamentos", quando comparado ao grupo de 7 a 13 anos, mostrando que o tratamento tem impacto mais negativo na percepção da QV dos adolescentes do que das crianças, o que também foi observado em outros estudos. ${ }^{19-22}$ A rotina rígida de tratamento diário interfere nas atividades e relações sociais dos adolescentes, sendo também, com frequência, o principal motivo pelo qual os colegas ficam sabendo da doença. ${ }^{18}$ Somam-se a isso a crescente complexidade do tratamento devido às comorbidades, ao agravamento da doença ao longo do tempo, ${ }^{22} \mathrm{e} o$ fato de que, na adolescência, o indivíduo começa a assumir, pelo menos parcialmente, a responsabilidade por seu tratamento, ${ }^{18}$ o que aumenta sua percepção sobre o impacto do mesmo.

Corroborando estudos anteriores, ${ }^{23}$ na percepção dos pais, o domínio "tratamentos" apresentou impacto significativamente maior na QV dos filhos do que na percepção das crianças menores de 14 anos, possivelmente porque nessa idade os pais ainda assumem a maior parte da responsabilidade pelo tratamento, ${ }^{18}$ o que contribui para a redução do impacto do mesmo na vida da criança.

Um estudo recente mostrou que a percepção materna a respeito do peso corporal do filho tende a ser distorcida, principalmente quando a criança apresenta excesso de peso ou obesidade, situações em que as mães tendem a subestimar o peso dos filhos. No entanto essa distorção também está presente, embora com menor frequência, entre mães de crianças com baixo-peso, que podem 
superestimar o peso dos filhos. ${ }^{24} \mathrm{O}$ presente estudo sugere que os responsáveis pelas crianças com FC têm consciência da dificuldade de ganhar peso gerada pela doença dos filhos. Talvez por isso subestimem o peso dos filhos, tendo em vista que o domínio "peso" foi o que recebeu a pontuação mais baixa na percepção dos pais, apesar de a maioria das crianças se apresentarem eutróficas no momento do estudo. Este resultado reflete a preocupação dos responsáveis com as deficiências nutricionais provocadas pela doença e com a dificuldade dos filhos para atingirem o peso ideal para a faixa etária. ${ }^{25-28} \mathrm{O}$ fato de a maioria dos pacientes se apresentarem eutróficos no momento da avaliação se deve possivelmente à atuação da equipe interdisciplinar, incluindo nutricionista e fisioterapeuta, que participam do acompanhamento de todos os pacientes do programa, como preconizado pelas diretrizes atuais de tratamento da $\mathrm{FC} .{ }^{25} \mathrm{Em}$ outro estudo brasileiro, realizado no estado de São Paulo, o domínio "peso" também recebeu a pontuação mais baixa na percepção dos pais. ${ }^{19}$

São frequentes sentimentos de culpa, impotência e vergonha relacionados à magreza dos filhos, em mães de crianças desnutridas. ${ }^{29}$ Contudo, não foram encontrados estudos sobre os sentimentos das mães de pacientes com FC, relacionados ao baixo-peso dos filhos, sendo este um campo para futuras investigações, considerando que o presente estudo mostrou que, na percepção dos responsáveis, o peso exerce grande impacto negativo sobre a QV dos filhos.

Os pacientes que se declararam negros ou pardos apresentaram pontuações significativamente menores com relação ao domínio "imagem corporal", quando comparados aos indivíduos que se declararam brancos. Um estudo norte-americano mostrou que crianças e adolescentes com FC, de origem afro-americana e hispânica, apresentavam menores escores nos domínios "físico", "social", "imagem corporal", "saúde" e "papel social". ${ }^{30}$ Não foram encontrados outros estudos brasileiros que tenham avaliado, em indivíduos com FC, o impacto da raça/cor nos domínios da QV. No entanto, um estudo com estudantes universitárias negras, com transtornos alimentares, mostrou que, nessas mulheres, a imagem corporal é influenciada negativamente pela vivência da discriminação racial e da pressão externa para se adequar a um padrão estético concebido a partir das características físicas das mulheres brancas. ${ }^{31}$ É provável que os resultados observados no presente estudo com relação a raça/cor estejam relacionados a fatores semelhantes, devido aos estereótipos de beleza impostos pela sociedade, baseados nas características físicas dos indivíduos da raça branca. ${ }^{32}$

Observou-se correlação positiva com força moderada entre o domínio "respiratório" e pIMC/I, indicando que um menor pIMC/I está associado a um maior impacto dos sintomas respiratórios na percepção da QV desses indivíduos. Embora esta relação não tenha sido observada em outros estudos realizados com adolescentes e adultos, ${ }^{22}$ está bem estabelecido na literatura que o estado nutricional tem relação com a severidade do comprometimento da função pulmonar em pacientes com FC..$^{26-28,33}$ 
Corroborando outros estudos, ${ }^{22,34}$ foi observada correlação positiva forte entre o domínio "imagem corporal" e o pIMC/I, indicando que quanto menor é o peso, pior é a imagem corporal. Esse resultado merece atenção, tendo em vista que "imagem corporal" e "peso" foram os dois domínios com pior pontuação, conforme discutido em parágrafos anteriores, o que significa que são os que apresentaram impacto mais negativo na QV das crianças e adolescentes com FC, de acordo com sua própria percepção, e também na percepção de seus responsáveis, no caso do domínio "peso".

É consenso na literatura que a colonização precoce por $P$. aeruginosa tem impacto significativo no prognóstico dos pacientes com FC, ${ }^{35}$ sendo importante preditor de morbidade e mortalidade. ${ }^{36}$ Contudo, apesar de a maioria dos participantes do estudo ser colonizado por bactérias, não foram encontradas diferenças entre os pacientes colonizados e os não colonizados com relação aos domínios da QV. É provável que isso se deva ao quadro de estabilidade clínica dos pacientes avaliados, pois esse resultado corrobora outro estudo, que avaliou o impacto da colonização por $P$. aeruginosa, no domínio "respiratório" do CFQ, em 100 pacientes com FC, clinicamente estáveis, e não mostrou diferença significativa entre os pacientes colonizados e os não colonizados. ${ }^{37}$

Não se observou correlação entre o Escore de Shwachman e a QV, o que difere do estudo de Cohen et al..$^{19}$ É importante ressaltar que, no presente estudo, 14 indivíduos (73,9\%) foram classificados como "excelente" ou "bom" de acordo com esse escore, o que possivelmente pode explicar a ausência de correlação, devido ao número reduzido de indivíduos nas categorias leve $(\mathrm{n}=4)$ e moderado $(\mathrm{n}=1)$, e à ausência de indivíduos classificados como graves.

Cinco pacientes deste estudo relataram tabagismo passivo. Entretanto, não houve diferença na QV desses indivíduos em comparação com o grupo cujos responsáveis não eram tabagistas. Esses dados corroboram um estudo recente realizado na Hungria, no qual o tabagismo passivo não teve impacto na QV das crianças com FC. ${ }^{38}$ Não foram encontrados outros estudos que tenham avaliado o impacto do tabagismo passivo na QV das crianças ou adultos com FC, mas é importante ressaltar que tem sido bem documentado o impacto negativo do tabagismo passivo na função pulmonar desses indivíduos. ${ }^{39}$

As duas principais limitações do presente estudo foram a impossibilidade de avaliar variações longitudinais, por tratar-se de um estudo transversal, e o número reduzido da amostra, que impossibilitou a avaliação de outras categorizações. Contudo, devemos considerar que, segundo o Registro Brasileiro de FC, ${ }^{40}$ o estado do ES tem um total de 135 pacientes cadastrados e em tratamento para FC, incluindo adultos e crianças, e o estudo foi realizado no único centro de referência pediátrico do ES para o tratamento da FC, que tinha no período analisado, 49 pacientes cadastrados na faixa etária estudada. Assim, embora pequena, a amostra corresponde a aproximadamente $39 \%$ dos indivíduos com FC do ES nessa faixa etária. 


\section{Conclusões}

A correlação positiva forte entre o pIMC/I e o domínio "imagem corporal” sugere que o baixo-peso tem impacto negativo na imagem corporal das crianças e adolescentes com FC. Esse resultado merece atenção, tendo em vista que "imagem corporal" e "peso" foram os domínios que mais afetaram negativamente a QV na percepção dos pacientes, e também na percepção dos pais, no caso do peso.

O pIMC/I apresentou correlação positiva moderada na percepção da QV com relação ao domínio "respiratório", evidenciando que, nos pacientes com menor peso, existe uma percepção mais acentuada do impacto negativo dos sintomas respiratórios na QV.

Raça/cor também teve impacto no domínio imagem corporal. Além disso, foi evidenciado que o impacto do tratamento na QV aumenta com a idade, na faixa etária estudada; que a "alimentação" tem maior impacto entre as crianças do que entre os adolescentes, embora seja o domínio com a melhor pontuação geral; e que na percepção dos pais, o domínio tratamento tem maior impacto na QV do que na percepção das crianças.

Esses resultados enfatizam a necessidade de que sejam otimizadas, priorizadas e inseridas, no SUS, estratégias para acompanhamento e correção de deficiências nutricionais e para o ganho de massa muscular em indivíduos com FC, com a participação da equipe multiprofissional, incluindo nutricionistas, fisioterapeutas e educadores físicos que atuam nessas áreas.

É importante que outros estudos sobre o assunto sejam realizados, nas diferentes regiões do país, com amostras maiores e avaliação longitudinal, para que se possa atuar de forma cada vez mais efetiva na melhora da QV desta população, tendo em vista que o Brasil apresenta importantes diferenças culturais, sociais, educacionais e étnicas, que podem interferir na percepção da imagem corporal e da QV.

\section{Colaboradores}

da Silva LA participou da elaboração do projeto, coleta de dados, análise de dados e redação do artigo; Lima ACP participou da elaboração do projeto, coleta de dados, análise de dados e redação do artigo; Wittmer VL contribuiu para a elaboração do projeto, análise de dados, redação e revisão do artigo; FMG Liberato FMG contribuiu para a redação do projeto, supervisionou a coleta de dados e contribuiu para a redação e revisão final do artigo; Arpini LSB contribuiu para a análise dos dados, redação e revisão final do artigo; Paro FM participou da elaboração do projeto, orientou a coleta e a análise dos dados e contribuiu na redação do artigo e revisão final.

Conflito de interesses: Os autores declaram que não haver conflito de interesses. 


\section{Referências}

1. Kerem B, Rommens J, Buchanan J, Markiewicz D, Cox T, Chakravarti A, et al. Identification of the cystic fibrosis gene: genetic analysis. Science. 1989;245(4922):1073-1080.

2. Riordan JR, Rommens JM, Kerem B, Alon N, Rozmahel R, Grzelczak Z, et al. Identification of the cystic fibrosis gene: cloning and characterization of complementary DNA. Science. 1989; 245(4922):1063-1063.

3. Ribeiro J, Ribeiro M, Ribeiro A. Controvérsias na fibrose cística: do pediatra ao especialista. J. Pediatr (Rio J). 2002; 78:171-186.

4. Elborn JS. Cystic fibrosis. Lancet. 2016; 388:2519-2531.

5. Cystic Fibrosis Foundation Patient Registry. 2014 Annual data report to the Center Directors. Bethesda, MD: Cystic Fibrosis Foundation; 2015.

6. Dalcin PTR, Silva FAA. Fibrose cística no adulto: aspectos diagnósticos e terapêuticos. J Bras Pneumol. 2008; 34(2):107-117.

7. Quittner A, Buu A, Messer M, Modi A, Watrous M. Development and validation of the cystic fibrosis questionnaire in the United States. Chest. 2005; 128(4):2347-2354.

8. Gee L, Abbott J, Conway SP, Etherington C, Webb AK. Development of a disease specific health related quality of life measure for adults and adolescents with cystic fibrosis. Thorax. 2000; 55(11):946-954.

9. Abbott J, Hart A, Havermans T, Matossian A, Goldbeck L, Barreto C, et al. Measuring health-related quality of life in clinical trials in cystic fibrosis. J Cyst Fibros. 2011; 10:S82-S85.

10. Abbott J, Morton AM, Hurley MA, Conway SP. Longitudinal impact of demographic and clinical variables on health-related quality of life in cystic fibrosis. BMJ Open. 2015; 5(5):e007418.

11. Iskrov GG, Stefanov RS, Bastida JL, Linertová R, Moreno JO, Aguilar OS et al. Economic burden and health-related quality of life of patients with cystic fibrosis in Bulgária. Folia Medica. 2015; 57(1):56-64.

12. Santos CI, Ribeiro JD, Ribeiro AF, Hessel G. Análise crítica dos escores de avaliação de gravidade da fibrose cística: Estado da arte. J Bras Pneumol. 2004; 30(3):286-298.

13. Onis M, Onyango AW, Borghi E, Siyam A, Nishida C, Siekmann J. Development of a WHO growth reference for school-aged children and adolescents. Bull World Health Organization. 2007; 85:660-667.

14. Rozov T, Cunha MT, Nascimento O, Quittner AL, Jardim JR. Linguistic validation of cystic fibrosis quality of life questionnaires. J Pediatr (Rio J). 2006; 82(2):151-156.

15. Callegari J, Sidia M. Bioestatística: princípios e aplicações. Porto Alegre: Artmed; 2003.90 p.

16. Debska G, Cepuch G, Mazurek H. Quality of life in patients with cystic fibrosis depending on the severity of the disease and method of its treatment. Postepy Hig Med Dosw. 2014; 68:498-502.

17. Tibosch MM, Sintnicolaas CJ, Peters JB, Merkus PJ, Yntema JB, Verhaak CM, et al. How about your peers? Cystic fibrosis questionnaire data from healthy children and adolescents. BCM Pediatr. 2011; 11:86. 
18. Rocha KB, Moreira MC, Oliveira VZ. Adolescência em pacientes portadores de fibrose cística. Aletheia. 2004; 20:27-36.

19. Cohen MA, Ribeiro MAGO, Ribeiro AF, Ribeiro JD, Morcillo AM. Avaliação da qualidade de vida de pacientes com fibrose cística por meio do Cystic Fibrosis Questionnaire*. J Bras Pneumol. 2011; 37(2):184-192.

20. Martins MLB, Tonial SR, Gama MEA, Ribeiro TH, Ribeiro JM, Barbosa JMA. Consumo de alimentos entre adolescentes de um estado do Nordeste brasileiro. Demetra: Alimentação, Nutrição \& Saúde. 2014; 9(2):577-594.

21. São Pedro ND, Araújo MDPN, Freitas MDCS. Significados do comer no cotidiano de adolescentes: narrativas de estudantes com excesso de peso de uma instituição federal de ensino médio da cidade de Salvador, Bahia. Demetra: Alimentação, Nutrição \& Saúde. 2016; 11(3):847-861.

22. Habib ARR, Manji J, Wilcox PG, Javer AR, Buxton JA, Quon BS. A systematic review of factors associated with health-related quality of life in adolescents and adults with cystic fibrosis. Ann Am Thorac Soc. 2015; 12(3):420-428.

23. Groeneveld IF, Sosa ES, Pérez M, Fiuza-Luces C, Gonzalez-Saiz L, Gallardo C, et al. Health-related quality of life of Spanish children with cystic fibrosis. Quality of Life Research. 2012;21(10):1837-1845.

24. Friedrich RR, Antunes T, Schuch I. Percepção materna do estado nutricional de crianças em escolas públicas de Porto Alegre-RS. Demetra: Alimentação, Nutrição \& Saúde. 2016; 11(1):211-223.

25. Athanazio RA, Silva Filho LVRF, Vergara AA, Ribeiro AF, Riedi CA. Grupo de Trabalho das diretrizes brasileiras. Diretrizes brasileiras de diagnóstico e tratamento da fibrose cística. J Bras Pneumol. 2017; 43(3):219-245.

26. Barni GC, Forte GC, Forgiarini LF, Abrahão CLDO, Dalcin PDTR. Factors associated with mal nutrition in adolescent and adult patients with cystic fibrosis. J Bras Pneumol. 2017; 43(5):337-343.

27. Mauch RM, Kmit AHP, Marson FADL, Levy CE, Barros-Filho ADA, Ribeiro JD. Association of growth and nutritional parameters with pulmonary function in cystic fibrosis: a literature review. Rev Paul Pediat. 2016; 34(4):503-509.

28. Chaves CRMDM, Cunha ALPD, Costa ACD, Costa RDSSD, Lacerda SV. Nutritional status and body fat distribution in children and adolescentes with Cystic Fibrosis. Cienc. Saúde Coletiva. 2015; 20(11):3319-3328.

29. Magalhães MDLB, Machado MMT, Leite ÁJM, Carvalho AMP, Silva ACAT. "É assim, com vergonha de sair com ele": percepções de mães de crianças desnutridas, em um meio socialmente desfavorecido. Demetra: Alimentação, Nutrição \& Saúde. 2016; 11(4):917-931.

30. Quittner AL, Schechter MS, Rasouliyan L, Haselkorn T, Pasta DJ, Wagener JS. Impact of socioeconomic status, race and ethnicity on quality of life in patients with Cystic Fibrosis in the United States. Chest. 2010; 137(3):642-650.

31. Jesus Bittencourt L, Oliveira Nunes M. Transtorno alimentar em estudantes negras de Salvador: a relação com a imagem corporal. Demetra: Alimentação, Nutrição \& Saúde. 2017; 12(1):169-192.

32. Costa MF, Soares JC. Mulheres em revista: representação corporal no imaginário social. Demetra: Alimentação, Nutrição \& Saúde. 2016;11(Supl.):1171-1184. 
33. Chaves CRMDM, Britto JAAD, Oliveira, CQD, Gomes MM, Cunha, ALPD. Association between nutritional status measurements and pulmonary function in children and adolescents with cystic fibrosis. J. Bras. Pneumol. 2009; 35(5):409-414.

34. Forte GC, Barni GC, Perin C, Casarotto FC, Fagondes SC, Dalcin PDTR. Relationship between clinical variables and health-related quality of life in young adult subjects with cystic fibrosis. Respiratory Care. 2015; 60(10):1459-1468.

35. Silva Filho LVRF, Ferreira FDA, Reis FJC, Britto MCAD, Levy CE, Clark O, et al. Infecção por Pseudomonas aeruginosa em pacientes com fibrose cística: evidências científicas sobre o impacto clínico, diagnóstico e tratamento. J Bras Pneumol. 2013; 39(4):495-512.

36. Emerson J, Rosenfeld M, McNamara S, Ramsey B, Gibson RL. Pseudomonas Aeruginosa and other predictors of mortality and morbidity in young children with Cystic Fibrosis. Pediatr Pulmonol. 2002; 34:91-100.

37. Johnston E, O’Neill K, Tunney M, Reid A, Rendall J, Downey D, et al. Effects of Pseudomonas aeruginosa (PA) status on health related quality of life (HRQoL) in patients with cystic fibrosis. J Cyst Fibros. 2012; 11:139.

38. Bodnar R, Kadar L, Holics K, Ujhelyi R, Kovacs L, Bolbas K, et al. Factors influencing quality of life and disease severity in Hungarian children and young adults with cystic fibrosis. Italian J Ped. 2014; 40(1):50.

39. Ong T, Schechter M, Yang J, Peng L, Emerson J, Gibson RL, et al. Socioeconomic status, smoke exposure, and health outcomes in young children with Cystic Fibrosis. Pediatrics. 2017; 139(2):e20162730.

40. Grupo Brasileiro de Estudos de Fibrose Cística (GBEFC). Registro Brasileiro de Fibrose Cística (REBRAFC) 2015 [Internet]. [acesso em: 22 nov. 2017]. Disponível em: http://portalgbefc.org.br/ wp-content/uploads/2017/11/Registro2015.pdf

Recebido: 17 de janeiro de 2018

Revisado: 04 de maio de 2018

Aceito: 23 de maio de 2018 
